\title{
Can short lists facilitate the collection of data on diagnosis, intervention and presenting issue in community health and outpatient care services? Lisa Fodero* and Joe Scuteri
}

Address: 4409/93 Liverpool Street, Sydney, NSW, 2000, Australia

Email: Lisa Fodero* - lisa.fodero@ healthconsult.com.au

* Corresponding author

from 24th Patient Classification Systems International (PCSI) Working Conference Lisbon, Portugal. 8-II October 2008

Published: 27 November 2008

BMC Health Services Research 2008, 8(Suppl I):AI4 doi:I0.II86/I472-6963-8-SI-AI4

This abstract is available from: http://www.biomedcentral.com/I472-6963/8/SI/AI4

(c) 2008 Fodero and Scuteri; licensee BioMed Central Ltd.

\section{Introduction}

NSW Health is undertaking the community health and outpatient care information project (CHOCIP), which will develop a patient level data collection across all community health and outpatient care services in NSW. It is the largest project of its type ever attempted in Australia and, when complete, will result in the collection of some 25 million unit records describing the services provided in community health and outpatient care settings. A key CHOCIP sub-project is to develop short lists, in consultation with clinicians, that represent the 10-20 most common values in the data domain for the data elements diagnosis, intervention and presenting issue for each of the defined 130 service types in community health and outpatient care. The reason for the development of these short lists is to avoid the use of clinical coders to retrospectively code the three data elements.

\section{Methods}

The methodology consisted of three major processes. First, a range of classification systems was evaluated to assess their suitability as the underlying base for the short lists for the three data elements. Second, draft short lists were developed for the three data elements for each of the 132 service types (i.e., 396 short lists) by reviewing the literature, conducting initial consultation with clinicians, and collecting and analysing the available (coded) data. Third, the draft short lists were refined by working with the Clinical Advisory Groups (CAGs) formed specifically to advise on one or more of the 132 service types, as appropriate. The refinement method was a three round Delphi process, two rounds by mail and the third through a face-to-face meeting. The refined short lists will be incorporated into the CHOCIP Data Dictionary and the enterprise systems from which the collected data are to be drawn.

\section{Results}

Through review of classification systems, it became clear that no one system would be suitable as the base for the short lists across the 132 service types. The evaluation showed that, for hospital outpatient clinics, it was most logical to use ICD-10-AM and ACHI. This decision allowed for maximum continuity and consistency of the outpatient data to be collected with hospital inpatient data already collected, where diagnosis and intervention are coded using ICD-10-AM.

For community health services, the decision was more difficult. ICD-10-AM and ACHI was one candidate pair. Also, a classification system specifically for activities and interventions in community health services had been formulated as part of a major project to develop an enterprise system for community health; namely, the Australian Classification and Terminology of Community Health (CATCH). The other major candidate was ICPC-2 PLUS, which is primarily used in general practice settings in Australia. The analysis found that many of the intervention 
categories in $\mathrm{CATCH}$ were based on ACHI codes and that ICD-10-AM diagnosis codes were used in a number of services using the community health enterprise system. There was a strong desire to link admitted patient data to outpatient and community health data for the same journey (e.g., breast cancer), so it was decided to choose ICD10-AM and ACHI as the basis for the short lists in most community health settings (to allow tracking of diagnosis codes).

It was found that there was little data available on diagnosis, intervention and presenting issue within existing systems. Review of the available data assisted in the generation of some of the draft short lists, but the majority of the short lists had to be developed by working directly with the CAGs. It took considerable work to actively engaged clinicians in this process, but it was found possible to produce short lists for each of the 132 service types for the data elements diagnosis, intervention, and presenting issue using the three-round Delphi process.

The final refinement process (to be conducted early in 2009) will consist of a pilot test of the short lists in 15 services across NSW, covering off at least one service type in each of the 132 categories across community health and outpatient services. The pilot test will be conducted over a period of six weeks using predominately manual data collection. Results of the pilot test will be used to refine the short lists, which will then be included in the CHOCIP Data Dictionary and enterprise systems.

\section{Conclusion}

The project has demonstrated that is possible to develop abbreviated value data domains for the collection of the data elements diagnosis, intervention and presenting issue across community health and outpatient care settings. The value domains were shown to be relevant to each service type, largely because of the homogeneity of patients and services within each service type. The short lists will continue to be evolved and refined as the CHOCIP data collection commences. Importantly, the short lists facilitate the collection of data that address the important questions of why do patients present for services? (presenting issue); what is wrong with them? (diagnosis); and what services are provided to them? (intervention) without the need for specialised clinical coders. These data will be extremely valuable in planning, developing and funding community health and outpatient care services in NSW.

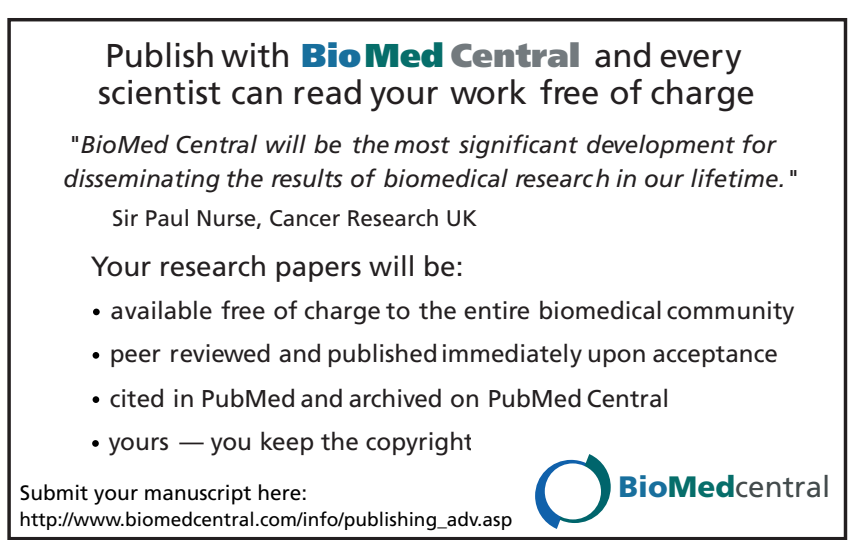

Page 2 of 2

(page number not for citation purposes) 